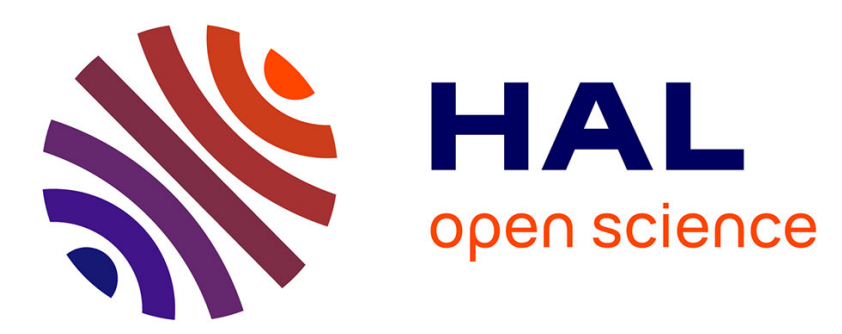

\title{
Medium-latitude sources of plasmaspheric nonthermal continuum radiations observed close to harmonics of the electron gyrofrequency
}

\author{
S. Grimald, Pierrette Décréau, P. Canu, A. Rochel, Xavier Vallières
}

\section{- To cite this version:}

S. Grimald, Pierrette Décréau, P. Canu, A. Rochel, Xavier Vallières. Medium-latitude sources of plasmaspheric nonthermal continuum radiations observed close to harmonics of the electron gyrofrequency. Journal of Geophysical Research Space Physics, 2008, 113 (A11), pp.A11216. 10.1029/2008JA013290 . insu-02871336

\section{HAL Id: insu-02871336 \\ https://hal-insu.archives-ouvertes.fr/insu-02871336}

Submitted on 17 Jun 2020

HAL is a multi-disciplinary open access archive for the deposit and dissemination of scientific research documents, whether they are published or not. The documents may come from teaching and research institutions in France or abroad, or from public or private research centers.
L'archive ouverte pluridisciplinaire HAL, est destinée au dépôt et à la diffusion de documents scientifiques de niveau recherche, publiés ou non, émanant des établissements d'enseignement et de recherche français ou étrangers, des laboratoires publics ou privés. 


\title{
Medium-latitude sources of plasmaspheric nonthermal continuum radiations observed close to harmonics of the electron gyrofrequency
}

\author{
S. Grimald, ${ }^{1,2}$ P. M. E. Décréau, ${ }^{1}$ P. Canu, ${ }^{3}$ A. Rochel, ${ }^{1}$ and X. Vallières ${ }^{1}$ \\ Received 8 April 2008; revised 24 July 2008; accepted 1 August 2008; published 22 November 2008.
}

[1] Nonthermal continuum (NTC) radiation is, with auroral kilometric radiation (AKR), one of the two electromagnetic emissions generated within the Earth's magnetosphere and radiated into space. It is generally believed that NTC is emitted in the plasmapause density gradient after conversion of intense electrostatic waves, present near the magnetic equator, into electromagnetic waves. In this paper, we present a specific type of NTC event, of infrequent occurrence, displaying a finger-like pattern not yet reported: banded emissions peaking at exact multiples of a common frequency, $\mathrm{df}$, which decrease inbound toward the plasmapause boundary layer (PPBL). Analysis is presented that indicates that the corresponding sources are nearby sites of the PPBL where the local electron gyrofrequency $f_{c e}$ equals df. The sources are radiating beams of limited cone angle size. The NTC sources for this event are shown to be located at about $20^{\circ}$ magnetic latitude. This illustrates that the PPBL is active in radiating NTC waves not only near the magnetic equator but also up to the medium-latitude range.

Citation: Grimald, S., P. M. E. Décréau, P. Canu, A. Rochel, and X. Vallières (2008), Medium-latitude sources of plasmaspheric nonthermal continuum radiations observed close to harmonics of the electron gyrofrequency, J. Geophys. Res., 113, A11216, doi:10.1029/2008JA013290.

\section{Introduction}

[2] Nonthermal continuum (NTC) radiation is an incoherent broadband electromagnetic radiation of low intensity and long duration which is observed in the frequency range from a few $100 \mathrm{~Hz}$ to several $100 \mathrm{kHz}$. It has been observed in the Earth environment [Gurnett, 1975; Etcheto et al., 1982; Morgan and Gurnett, 1991; Kasaba et al., 1998; Décréau et al., 2004] as well as in the environment of other magnetized planets [Kurth, 1992]. Terrestrial NTC is widely believed to be generated at the equatorial plasmapause, a region of strong density gradient, by conversion of electrostatic to electromagnetic waves. The electrostatic source has been suggested to be intense electrostatic waves, banded in frequency, found near the plasma frequency in the dawn outer plasmapause region, associated with $1-30 \mathrm{keV}$ electrons [Gurnett and Frank, 1976]. The spacing between narrow spectral features has been related to the gyrofrequency at the source [Kurth et al., 1981] by the relation:

$$
\mathrm{f}=\mathrm{f}_{\mathrm{UH}} \sim(\mathrm{n}+1 / 2) \mathrm{f}_{\mathrm{ce}}
$$

where $\mathrm{f}$, the frequency of $\mathrm{NTC}, \mathrm{f}_{\mathrm{UH}}$, the upper hybrid frequency, and $\mathrm{f}_{\mathrm{ce}}$, the electron cyclotron frequency, are

\footnotetext{
${ }^{1}$ Laboratoire de Physique et Chimie de l'Environnement, CNRS, Orleans, France.

${ }^{2}$ Now at Department of Space and Climate Physics, Mullard Space Science Laboratory, University College London, Surrey, UK.

${ }^{3}$ Centre d'Etude des Environnements Terrestre et Planétaires, Velizy, France.

Copyright 2008 by the American Geophysical Union. 0148-0227/08/2008JA013290
}

defined at the source. The implied underlying scenario is detailed by Gough [1982]. Near the plasmapause, plasma frequency decreases rapidly with increasing radial distance, crossing several successive electron gyroharmonic frequencies in only a short distance. It is assumed that strong electrostatic $\mathrm{f}_{\mathrm{UH}}$ waves are produced, offset in frequency from each harmonic by a similar amount. Conversion of these waves to electromagnetic waves yields a series of narrow-band continuum radiation features, separated in frequency by approximately the electron gyrofrequency, which is quasi-uniform within the equatorial plasmapause layer, at least in case of a narrow layer.

[3] There is experimental evidence that NTC sources are indeed located near the magnetic equator [Morgan and Gurnett, 1991], consistent with the observations of equatorial confinement of $\mathrm{f}_{\mathrm{UH}}$ electrostatic emissions [Gough et al., 1979]. However, Menietti and Yoon [2006] reported ordinary mode generation linked to density cavities, and located at medium magnetic latitude. Moreover, CLUSTER directivity analysis of NTC waves for a particular event (9 July 2001) has led to the detection of an NTC source placed at a large distance from the magnetic equator [Décréau et al., 2004]. In this event, associated with a steep plasmapause density gradient, NTC waves display a wideband spectral signature.

[4] In this paper, we present an interesting event similar to the event analyzed by Décréau et al. [2004], however with a richer spectral signature, reported here for the first time. The spectral signature is characterized by a series of frequency bands, peaking according to a harmonic pattern, $\mathrm{f}=\mathrm{n} \mathrm{f}_{\mathrm{ce}}$, in contrast to relation (1). Actually, several mechanisms, both direct [Farrell, 2001] and indirect [Yoon et al., 
1996, 1998; Weatherwax et al., 1995; Willes et al., 1998], developed to explain the generation of $\mathrm{O}$ mode at $\mathrm{f}=\mathrm{n} \mathrm{f}_{\mathrm{ce}}$ in the solar wind and in the ionosphere, could be at work here. All are based on the electron cyclotron maser instability. In this event, the dimension and the configuration of Cluster constellation allows us to identify the source position with good precision, confirming that the region of the plasmapause can radiate NTC waves from medium-latitude sources. Since no sharp boundary can be unequivocally identified as the plasmapause, this region will be denoted plasmapause boundary layer (PPBL) throughout this paper. We present the observations, and wave behavior derived from their analysis, in section 2 . Section 3 is devoted to the discussion of the frequency pattern and source positioning. Section 4 concludes.

\section{Observations and Data Analysis}

\subsection{Global Morphology of Waves}

[5] The Cluster mission consists of four identical satellites (which we denote here by $\mathrm{C} 1, \mathrm{C} 2, \mathrm{C} 3$ and $\mathrm{C} 4$ ), arranged in a tetrahedral configuration. They travel in a near-polar orbit with perigee located close to $\sim 4.5 \mathrm{R}_{\mathrm{E}}$, near the ecliptic. On the date considered, 30 December 2003, geomagnetic activity is low $\left(\mathrm{Km}=1^{\circ}\right)$, such that the constellation travels in the dipolar region for a couple of hours near perigee, which is placed at a local time of about 0430 MLT. During this time interval, inter-spacecraft separation distances are less than $0.25 \mathrm{R}_{\mathrm{E}}$ (see Figure $2 \mathrm{a}$ ).

[6] In this paper, the electronic cyclotron frequency $\left(f_{c e}\right)$ is derived from the analysis of Whisper active spectra, known to provide this value in good agreement, sometimes with better precision, with the magnetometer data [Décréau et al., 1997]. Observations presented in this paper are derived mainly from the WHISPER instrument. It is a relaxation sounder [Décréau et al., 1997, 2001] using for reception one of the two long double sphere antennas of the EFW instrument [Gustafsson et al., 1997]. The receiving antenna has a sphere-to-sphere separation of $88 \mathrm{~m}$ and rotates in the spin plane, which is parallel to the $\mathrm{X}_{\mathrm{GSE}}-\mathrm{Y}_{\mathrm{GSE}}$ plane, at a $4 \mathrm{~s}$ period. The wave form is acquired and a FFT performed every $13.33 \mathrm{~ms}$. Accumulated frequency spectra are delivered every 2 s [Décréau et al., 2001]. Sounding operations provide the electronic plasma frequency $\left(f_{\text {pe }}\right)$ and the electronic cyclotron frequency $\left(f_{c e}\right)$ at a recurrence of $52 \mathrm{~s}$ or $104 \mathrm{~s}$. Cross-calibration studies between measurements of FGM [Balogh et al., 2001] and WHISPER sounder indicate that both instruments measure exactly the same value of magnetic field amplitudes (within measurement errors).

[7] Figure 1a presents a frequency-time spectrogram of the electric field measured by WHISPER near perigee. C3 enters the plasmasphere at about 0847 UT in the southern hemisphere, and leaves it at about 1007 UT in the northern hemisphere. The phenomena of interest (pointed out by orange ovals) are visible outside both plasmapause boundaries. These boundaries are crossed by Cluster at medium magnetic latitudes, higher than $20^{\circ}$ in absolute value. These are NTC banded emissions (four bands inbound, two of them outbound), separated by a frequency interval of same order of magnitude as the respective values of the electron gyrofrequency $\mathrm{f}_{\mathrm{ce}}$ at each plasmapause crossing. The reso- nance at $\mathrm{f}_{\mathrm{ce}}$ and its harmonics (harmonic 4 pointed by a white arrow) are clearly visible during sounding operations, occurring at a $104 \mathrm{~s}$ interval during this perigee pass (see dots in Figure 1a).

[8] Figure 1b shows in greater details the NTC emissions in the southern hemisphere. They appear in the frequency range $30-60 \mathrm{kHz}$, above the plasma frequency $\mathrm{f}_{\mathrm{pe}}$ indicated by a solid white line. The emissions are structured in frequency bands (about $3 \mathrm{kHz}$ frequency width), their peak frequencies decreasing with time. The detailed spectrum measured at 0842:58 UT is shown on the right. The local plasma frequency $f_{p e}$ separates different wave patterns, visible respectively above and below $\mathrm{f}_{\mathrm{pe}}$. We identify the waves below $\mathrm{f}_{\text {pe }}$, peaking at $\sim(\mathrm{n}+1 / 2) \mathrm{f}_{\mathrm{ce}}$, as electrostatic waves commonly observed in the trough region [Hubbard and Birmingham, 1978]. The four bands above $\mathrm{f}_{\mathrm{pe}}$, which we interpret as electromagnetic NTC emissions, display a different spectral signature. The maximum amplitude of the emissions in each band is generally found near the lowfrequency cutoff of the band, and is decreasing in amplitude with increasing order of the band. From now on, we associate a number $\mathrm{n}$ to a given band, increasing from $\mathrm{n}=$ 1 for the band of lowest frequency $(\sim 30 \mathrm{kHz})$, to $\mathrm{n}=4$ for the band of highest frequency. Further analysis of the spectral pattern is presented in section 2.2.

[9] Figure 1c displays a detailed spectrogram at the inbound plasmapause boundary. In Figure 1c, the wave intensity is expressed in $\mathrm{dB}$ above $10^{-7} \mathrm{~V}_{\mathrm{rms}} \cdot \mathrm{Hz}^{-1 / 2}$. Resonance peaks triggered by sounders operations appear at dots at regular time spacing. The two consecutive sounding operations placed before and after 0848 UT (white arrows), provide the gyrofrequency $f_{c e}$ and the plasma frequency $f_{p e}$ on both sides of the main density jump. Between sounding, $\mathrm{f}_{\mathrm{pe}}$ is derived from natural emissions signatures [Canu et al., 2001]. The gyrofrequency measured by the WHISPER instrument $(\sim 7.80 \mathrm{kHz})$ is almost constant, whereas the measured plasma frequencies (28 and $54 \mathrm{kHz}$, successively) differ by $26 \mathrm{kHz}$, corresponding to a density jump $\Delta \mathrm{n} \sim 16 \mathrm{~cm}^{-3}$. We note that, in contrast with Gough's [1982] view of an equatorial source region, the PPBL crossing observed here is located at a medium latitude of $\lambda_{\mathrm{m}} \sim 20^{\circ}$, nevertheless encountered at the same magnetic latitude of $\lambda_{\mathrm{m}} \sim 20^{\circ}$ for all spacecraft. The gyrofrequency is thus still essentially constant inside a constant latitudinal slice of the-PPBL, in accordance with Gough's view (then generalized at nonequatorial latitudes).

\subsection{Analysis of NTC Beams in the Southern Hemisphere}

[10] We focus now on the behavior of NTC bands observed in the Southern Hemisphere, between 0827 and 0846 UT by C3 (Figures 1a and 1b), and at similar time intervals by the other spacecraft. Near perigee, the Cluster tetrahedron is elongated (Figure 2a). The inter-spacecraft separation distance ranges from $\sim 300 \mathrm{~km}(\mathrm{C} 2-\mathrm{C} 3)$ to $1200 \mathrm{~km}(\mathrm{C} 1-\mathrm{C} 4) . \mathrm{C} 1$ is leading, and then comes the pair $\mathrm{C} 3$ and $\mathrm{C} 2, \mathrm{C} 4$ brings up the rear. In order to compare signal amplitudes observed from the different spacecraft, we selected a specific frequency, $39.5 \mathrm{kHz}$, pinpointed by the dashed line in the spectrogram displayed in Figure 1b. After $0840 \mathrm{UT}$, that frequency belongs to the second harmonic band (in order of increasing frequencies). Time variations of 

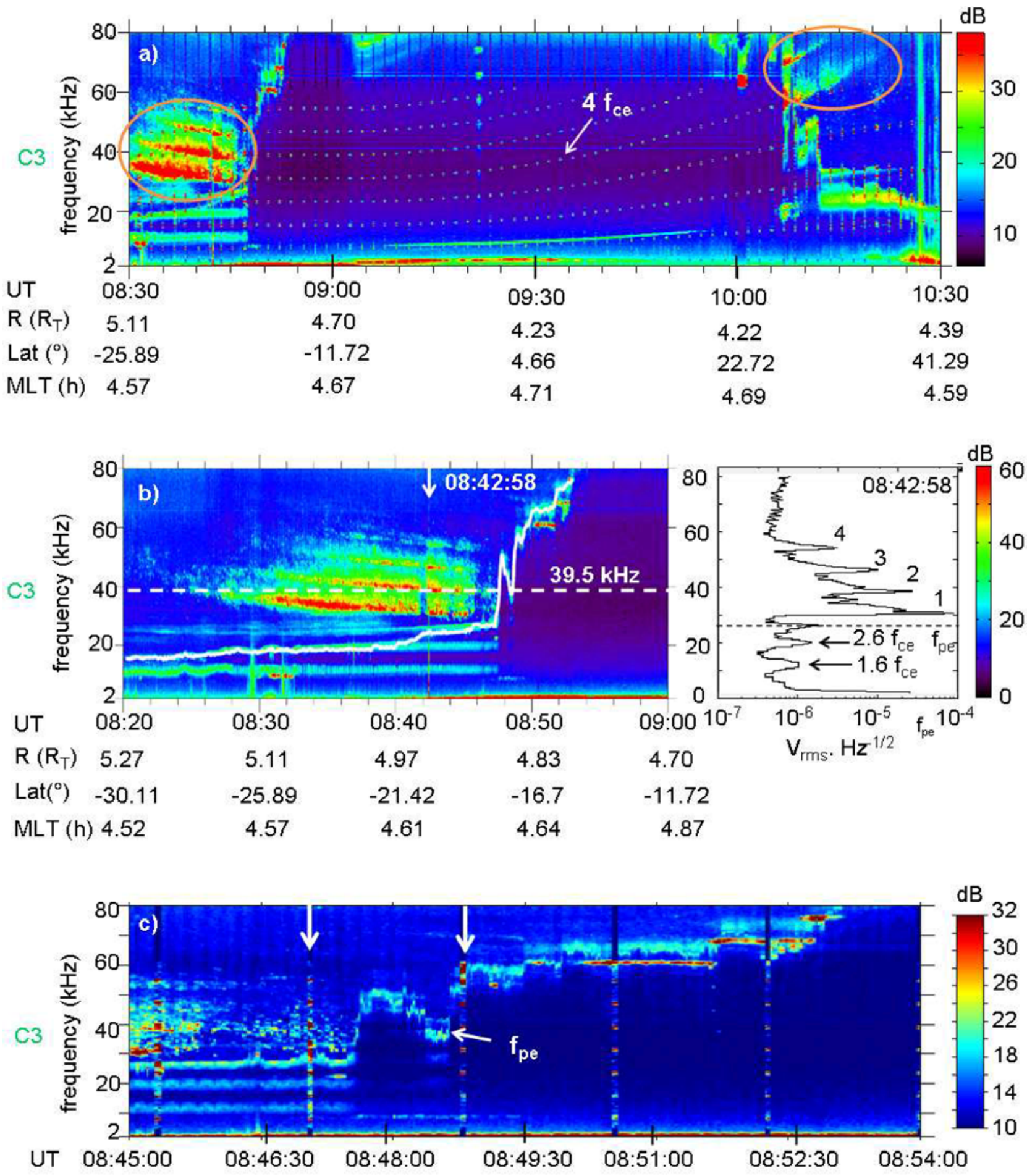

Figure 1. Wave spectral signatures at the plasmasphere crossing event of 30 December 2003 observed by C3 Cluster satellite. (a) Frequency/time spectrogram of the complete plasmasphere crossing. Wave intensity is reported in $\mathrm{dB}$ above $10^{-7} \mathrm{~V}_{\mathrm{rms}} . \mathrm{Hz}^{-1 / 2}$. Resonance peaks triggered by sounder operations, in particular at $\mathrm{f}_{\mathrm{ce}}$ and harmonics, appear as dots at regular time spacing. Orange ovals point to the phenomenon of interest (wideband NTC band emissions). In the two cases, inbound toward the plasmapause as outbound from it, the PPBL crossing is observed at medium magnetic latitude and NTC band spacing in close to local $f_{\text {ce. }}$ (b) (left) Frequency/time spectrogram centered on southern NTC waves. The white line indicates the position of plasma frequency $\left(\mathrm{f}_{\mathrm{pe}}\right)$. The NTC band spacing at the PPBL crossing $(\approx 0845$ UT) is close to the local $f_{\text {cee }}$. (right) Frequency spectrum measured at $\sim 0843$ UT. The plasma frequency $\left(f_{\text {pe }}\right)$ separates the electrostatic waves observed at $(n+1 / 2) f_{c e}\left(\right.$ below $\left.f_{p e}\right)$ and the NTC electromagnetic waves (above $f_{\text {pe }}$ and numbered from 1 to 4). (c) Details of the inbound plasmapause crossing shown in Figure 1b. Arrows point to sounder operations on both sides of the main density jump. 

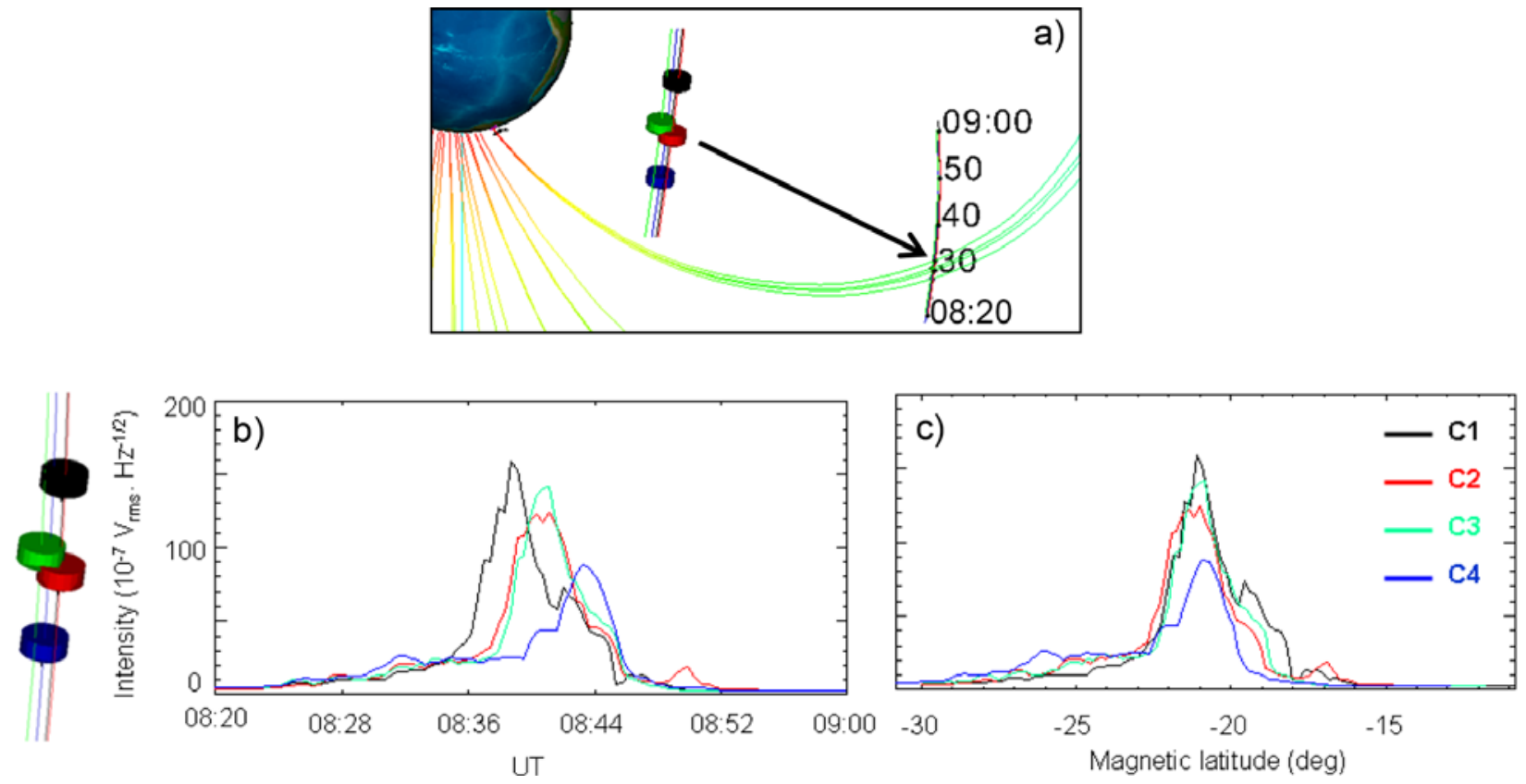

Figure 2. (a) Orbit of the satellites and constellation configuration at 0830 UT (produced with Orbit Visualization Tool http://ovt.irfu.se)). (b) Temporal and (c) latitudinal profiles of electric field amplitude measured from the four satellites at $39.5 \mathrm{kHz}$. Latitudes are expressed in SM coordinates. The color coding is shown on the right, and the constellation configuration at 0830 UT is shown at the left. All satellites display a similar behavior, encountering the amplitude growth in exactly the same order of spacecraft along the line of pearls configuration and at the same magnetic latitude. This suggests a stable beam of limited cone angle illuminating part of Cluster orbit.

electric field amplitudes at $39.5 \mathrm{kHz}$ are displayed in Figure $2 \mathrm{~b}$, color codes referring to the various satellites (black: C1, red: $\mathrm{C} 2$, green: $\mathrm{C} 3$, blue: $\mathrm{C} 4$ ). As expected from Figure $1 \mathrm{~b}$, the amplitude grows, reaches a maximum when the second harmonic band is encountered, then fades away. It is striking that all satellites display a similar behavior, peaking times arranged in the order $\mathrm{C} 1, \mathrm{C} 2$ and $\mathrm{C} 3$, then $\mathrm{C} 4$, the exact ordering of spacecraft along the line of pearls configuration. This suggests a scenario where a stable beam, of limited cone angle, is illuminating just a part of Cluster orbit, reached by the different spacecraft one after another, and crossed within a few minutes. This hypothesis is confirmed by the plot of Figure 2c, displaying amplitude profiles versus latitude. All peaks are observed at the same latitude. The fact that $\mathrm{C} 4$ sees a somewhat lower amplitude than the other spacecraft can be interpreted by a more detailed analysis, taking into account the limited size of the cone angle and the presence of shadowing by the multiple density irregularities observed by the four spacecraft in this region (not shown here). A visible fading from such a density irregularity can be seen for instance by C3 at 0843 UT (Figure 1b). The time interval during which the beam can be considered as stable is at least $10 \mathrm{~min}$ according to the time delay between the first and last spacecraft passages.

[11] The tight frequency link between the NTC bands observed at a given time by a given satellite suggests that they are emitted from a common narrow source region, where a local characteristic frequency operates as a harmonic filter. Narrow source regions for NTC have already been reported from Whisper observations close to the plasmapause [Canu et al., 2006]. Directivity analysis, not presented in detail in this paper, confirms this view. In brief, we applied the directivity tools presented by Décréau et al. [2004], making use of the spin modulation of the measured electric field amplitude. This yields a frequency-time spectrogram of the direction of the raypaths in the spin plane, a plane parallel to the $\mathrm{X}-\mathrm{Y}$ plane (GSE coordinates). According to this analysis, raypaths linked to all spectral peaks observed at a given time are aligned with each other, pointing toward the Earth. The directivity analysis indicates thus that all frequency bands behave similarly, pointing to a common source region.

[12] In order to further study the link between the different bands, observed simultaneously, we considered the frequency position of the main peaks, noted peak 1 to 4 in each individual frequency spectrum (Figure $1 \mathrm{~b}$, right). Those values, labeled $f_{1}$ to $f_{4}$, are plotted versus the magnetic latitude in Figure 3a, for spacecraft C3. Differences in frequency between two consecutives peaks, $d f=f_{i}+1-f_{i}$, are plotted versus latitude in Figure 3b. They behave similarly, the dashed black curve showing the variation trend with latitude of df. The dashed black curve has been obtained from linear regression applied on all df (lat) measurements. Figure 3 a displays, superposed on the observed peak frequencies, plots of multiples of this common frequency df (dashed lines), demonstrating that the peaks are indeed presenting a common harmonic pattern at any given time. A similar behavior is noted when analyzing spectra from other spacecraft, as well as when analyzing low-frequency cutoffs of the two bands showing up in the Northern Hemisphere. For this particular 

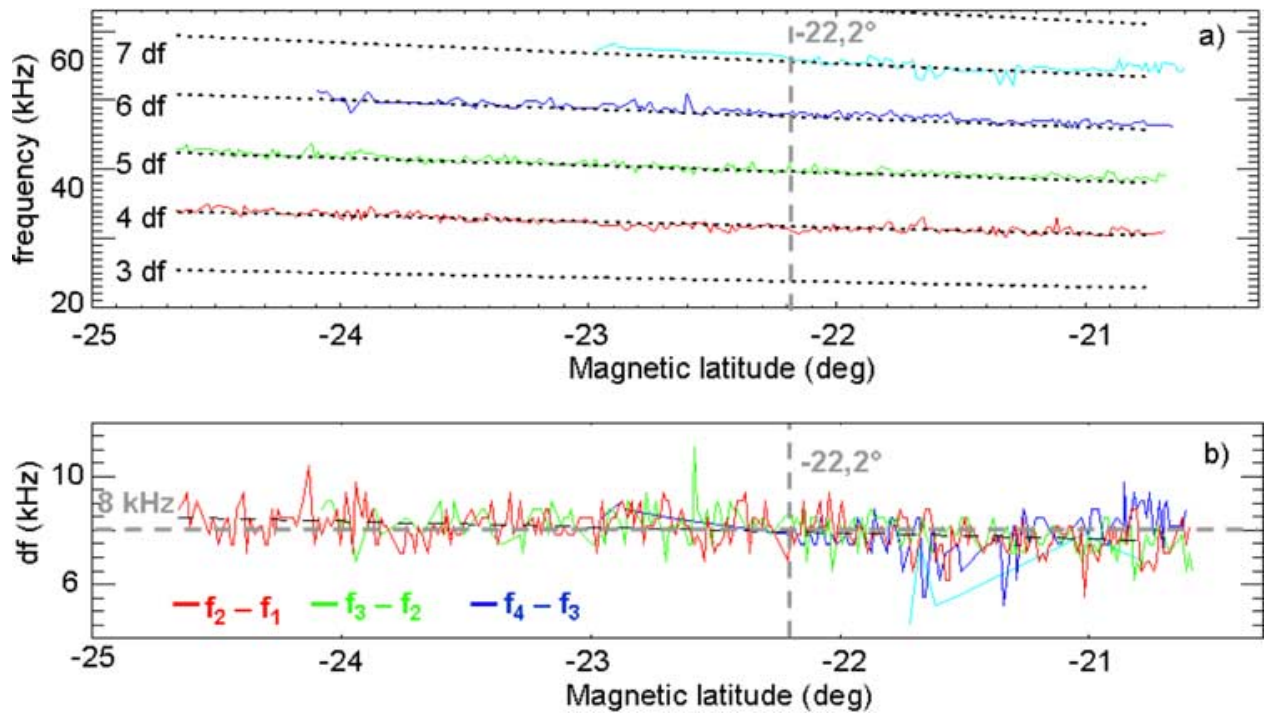

Figure 3. (a) Evolution with latitude of NTC peak frequencies observed on satellite $\mathrm{C} 3$ and (b) differences in frequency between various pairs of consecutives peaks. Those differences behave similarly, the dashed black line giving the variation trend $\mathrm{df}$. The black dotted lines in Figure 3a give the multiples of $\mathrm{df}$ and show that frequency peaks appear at exact multiples of $\mathrm{df}$.

event, the frequency peaks satisfy $\mathrm{f}=\mathrm{n}$ df on all spacecraft, on both sides of the plasmasphere.

\section{Discussion}

\subsection{Frequency Pattern Near the Source}

[13] Having established that spectral peaks of the banded NTCs are organized as multiples of the same frequency at any given time, it is interesting to discuss what this common frequency df means. Figure 1a indicates that the value df is, in both hemispheres, of the same order of magnitude as the electron gyrofrequency measured respectively at each closest plasmapause crossing. It is thus natural to consider the hypothesis where df equals a nearby local gyrofrequency. Assuming this is the case, and accepting, with Gough [1982], the relation $\mathrm{f}=\mathrm{f}_{\mathrm{UH}}=(\mathrm{n}+\delta) \mathrm{f}_{\text {ce }}$, where $0<\delta<1$ and where $\mathrm{n}$ is an integer $\geq 1$, leads to a very small value of $\delta$, at odds with the event case reported by Kurth et al. [1981], where $\delta \sim 0.5$.

[14] Another possible option would be to assume with Rönnmark [1985] that local Bernstein waves $\left(\mathrm{f}_{\mathrm{qn}}\right)$ are generating NTC, via a decay mechanism. This could possibly generate, under a given plasma regime (homogeneous layer at $\mathrm{f}_{\mathrm{pe}} \sim$ constant), a series of quasi-harmonic waves. Under that assumption, the frequency separation $f_{q(n+1)}-f_{q n}$ would however not be constant. We shall thus stick to Gough's view, where each frequency of the $(n+\delta) f_{\text {ce }}$ series is emitted for a different plasma frequency, i.e., at different positions inside the PPBL. Examples of narrow band intense emissions at multiples of local $f_{\text {ce }}$ are seen in Figure 1c from about 0850 to 0853 UT. Those emissions appear in the frequency range covered by the plasma frequency during the event (between 60 and $80 \mathrm{kHz}$ ). Such emissions are probably electrostatic waves and possible sources of NTC (not visible in the spectrogram, the satellite being too close to the source). Similar intensifications are observed on the northern flank of the plasmapause (Figure 1a, 0957 - 1007 UT). Since the plasma frequency profile is very irregular, it is not possible, owing to limited frequency and time resolutions, to identify precisely which characteristic frequency $\left(\mathrm{f}_{\mathrm{UH}}\right.$ or $\mathrm{f}_{\mathrm{qn}}$ ) is intensified for this example. We will in any event consider that radiated frequencies are created in the PPBL, where electrostatic waves are intensified, and where the outside low density of the plasmatrough allows the converted electromagnetic radiation to escape.

[15] A third option is that Cluster is observing narrow band electromagnetic emissions generated very near to the exact harmonic of electron gyro frequency and theoretically predicted, as indicated in the introduction. For instance, the mechanism proposed by Farrell [2001] and Farrell et al. [2004] relies upon the Electron Cyclotron Harmonic maser emissions triggered by mildly energetic electrons, commonly present in the PPBL.

[16] Assuming that the NTC sources have a frequency which is a multiple of the local electron gyrofrequency as suggested by the above example allows deriving their location with precision. This can be illustrated by simple quantitative considerations, illustrated in Figures $4 \mathrm{a}$ and $4 \mathrm{~b}$. Figure $4 \mathrm{~b}$ presents the configuration of three iso- $\mathrm{f}_{\mathrm{ce}}$ lines ( $7 \mathrm{kHz}$ in blue, $8 \mathrm{kHz}$ in red and $9 \mathrm{kHz}$ in purple) in a meridian plane in SM coordinates. The $f_{c e}$ value increases when geocentric distance decreases. The Cluster $\mathrm{C} 3$ orbit is plotted in green. If the source region is placed between the spacecraft and the Earth, as indicated by the directivity analysis reported in 2.2 , then the df value must be higher than $f_{\text {ce }}$ measured at the spacecraft. This is the case, as illustrated in Figure 4a which compares the gyrofrequency $f_{c e}$ measured at the spacecraft $\mathrm{C} 3$ with df value measured simultaneously. Both values are converging when $\mathrm{C} 3$ meets the PPBL, consistent with the fact that sources are in the PPBL region. This allows model source positions on basis of the assumption that $\mathrm{df}=\mathrm{f}_{\mathrm{ce}}$ at the source, as described below.

\subsection{Position of the NTC Sources}

[17] We have constructed a diagram positioning the radio sources at intermediate scale, i.e., where each source 

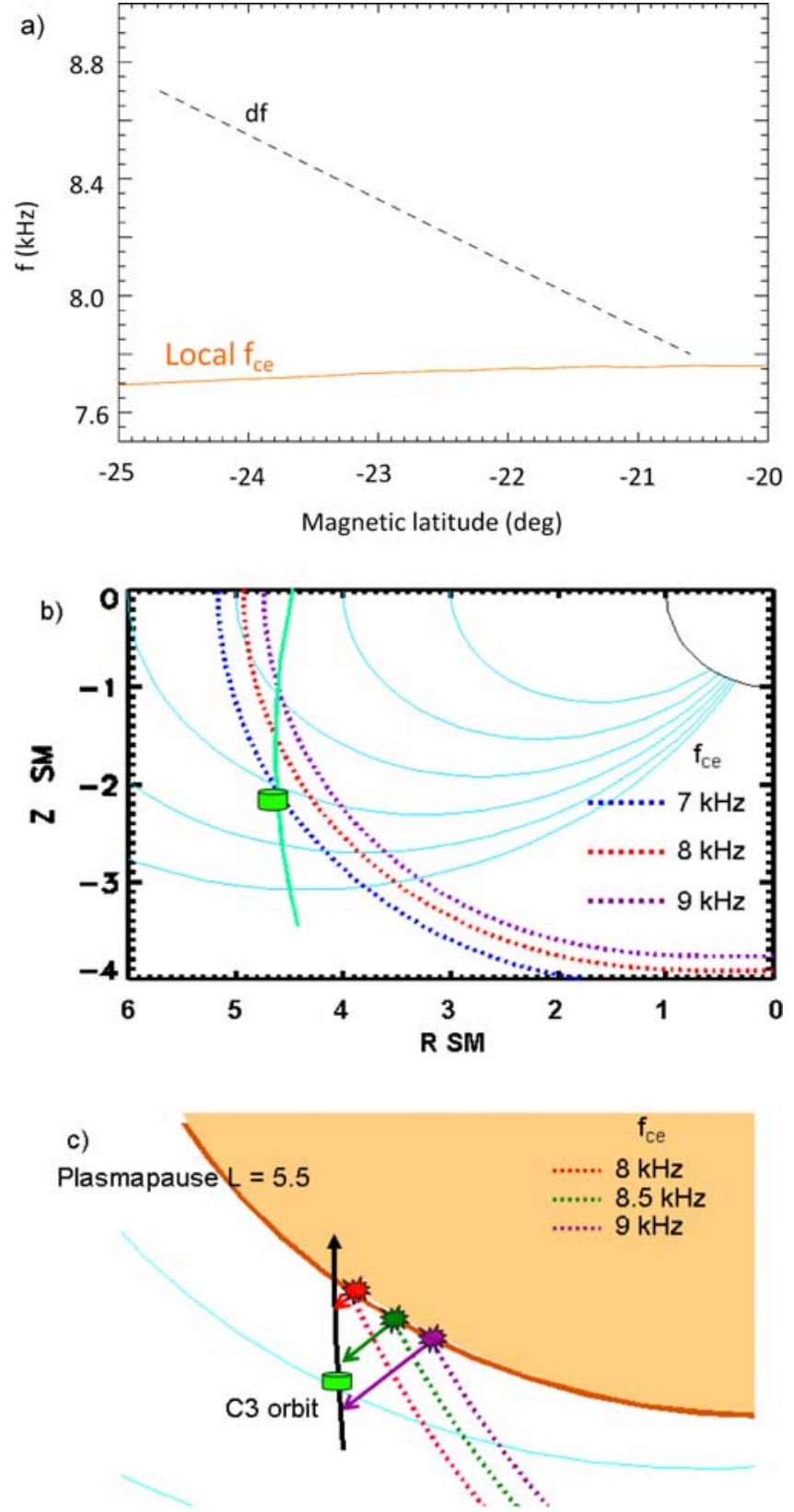

Figure 4. Localization of NTC sources in a meridian plane. (a) Evolution versus latitude of local gyrofrequency $\left(\mathrm{f}_{\mathrm{ce}}\right)$ and of the evolution of average harmonic distance (df) of observed bands plotted in Figure $3 \mathrm{~b}$. The local $\mathrm{f}_{\mathrm{ce}}$ and $\mathrm{df}$ are converging when C3 meets the PPBL. (b) Magnetic shells and iso- $f_{\text {ce }}$ surfaces. C3 orbit is in green; $f_{\text {ce }}$ decreases with geocentric distance. In agreement with Figure $3 \mathrm{a}$ and under the assumption that sources generate waves at local $\mathrm{f}_{\mathrm{ce}}$, the sources get closer to a satellite when it approaches the PPBL. (c) Interpretive diagram (zoom of Figure 4b) displaying wave sources and their central beams.

corresponds to an ensemble of micro-sources responsible for the series of banded frequency beams at given $f=n d f$ (emitted by a given iso- $\mathrm{f}_{\mathrm{ce}}$ cell encompassing the entire PPBL). The model, presented in Figure 4c, is based on two main assumptions: (1) that sources are placed in the strong gradients found close to the PPBL and (2) that their center belongs to the iso-fce surface $f_{c e}=d f$. A third property, derived from directivity analysis (raypaths associated with frequency peaks pointing toward the Earth) indicates that sources of spectral peaks are placed in the meridian plane containing the Cluster constellation. The plasmapause layer is supposed to lie between two $\mathrm{L}$ shells encountered at $\mathrm{L} \sim 5.5$ at the inbound Cluster crossing. The intersection of the central $\mathrm{L}$ shell with the iso- $\mathrm{f}_{\mathrm{ce}}$ surface at a given $\mathrm{f}_{\mathrm{ce}}$ uniquely defines the iso- $f_{\text {ce }}$ cell emitting the band series at $d f=f_{\text {ce }}$. Since $f_{\text {ce }}$ values measured on board behave like those of a dipole model (latitudinal and radial variations) but are a few percent lower than modeled ones, the iso- $\mathrm{f}_{\mathrm{ce}}$ shown in Figure 4 are derived from a dipole model where the reference magnetic field amplitude has been multiplied by a constant factor, such that modeled values fit measured ones. Figure $4 \mathrm{c}$ illustrates the positions and dimension of the three source regions derived from this scheme, at $\mathrm{f}_{\mathrm{ce}}$ values respectively $8,8.5$ and $9 \mathrm{kHz}$. Their size, $\sim 300 \mathrm{~km}$ radius, matches the typical density jumps near the plasmapause quite well.

[18] The observations clearly establish that these emission beams are produced well off the magnetic equatorial plane, at about $-22^{\circ}$ or $-23^{\circ}$ magnetic latitude. The scheme of sources at intersection of strong density gradient at iso- $\mathrm{f}_{\mathrm{ce}}$ gives an indication about the angular width of a beam issued from a given source region (from a given frequency). This indication can be derived from the length of the illuminated orbit element (at $\mathrm{f}=39.5 \mathrm{kHz}$, a $1.5-2^{\circ}$ range of latitude is obtained from Figure 2c) compared to the distance of the source to the orbit (Figure 4c). Both distances are of the same order of magnitude (about $0.1 \mathrm{R}_{\mathrm{E}}$ ) leading to a large angular global width.

[19] Arrows featuring raypaths are drawn from each source region up to the position of Cluster when it observes NTC at matching frequencies (spectral peaks at $\mathrm{df}=\mathrm{f}_{\mathrm{ce}}$ frequency distance). This exercise provides a coherent picture, where central raypaths are roughly parallel to each other, each fraction of the orbital path $(\sim 4.000 \mathrm{~km}$ total $)$ being exposed mainly to a beam ensemble of a given harmonic pattern. We observe that the highest frequencies are radiated from the most distant sources, in accordance with the fact that the corresponding amplitudes are the faintest.

\section{Conclusion}

[20] The Cluster constellation provides new capabilities in observing radio waves, in particular, (1) a multipoint view and (2) the spectral analysis of a large frequency band at a time resolution sufficient to resolve spatial features of $\sim 10 \mathrm{~km}$ dimension. Such capabilities can be taken advantage of while observing NTC radio waves in the outer plasmasphere region. A detailed analysis of the spectral properties of NTC waves, measured at successive orbital positions while approaching the plasmasphere on 30 December 2003, leads to the interpretive picture displayed in Figure 4c. Several NTC sources, placed along the plasmapause layer at medium latitudes, are radiating beams of limited angular size intercepted by nearby Cluster observatories. This event is not unique. We have studied other events of the same type which confirm this picture. One is observed on the same orbit, outbound from the plasmasphere crossing, from a view point placed at a similar L value, but lower altitude. 
[21] Those results open new perspectives on two main aspects of NTC waves. The first aspect is that the plasmapause layer radiates NTC waves, not only from a region concentrated toward the magnetic equator, but from a much larger part of the magnetic shell. Observed wave intensities are moderate, not far from electronic noise level when the distance to the plasmapause is significant, which can explain why those sources have been previously ignored. The second aspect of importance is that each latitudinal slice of the plasmapause layer radiates within a small beaming cone angle. The size of half cone angle is of order 2 degrees or less, as estimated from variation of the peak frequency with satellite position, compared to the distance to modeled source. We have evidenced also that narrow band emissions can be generated at frequencies very close to $\mathrm{nf}_{\mathrm{ce}}$. This does not question the standard interpretation of NTC emissions produced close to $\mathrm{f} \sim(\mathrm{n}+1 / 2) \mathrm{f}_{\text {ce }}$. It only shows that several mechanisms could lead to the generation of NTC. These facts are interesting information for any future studies of the NTC generation mechanism. No conclusion can be drawn on the question of whether midlatitude NTC sources are created by the same processes as equatorial ones (the processes are yet to be explained, in both situations). Models will have to take into consideration the large density irregularities observed in the plasmapause layer for most events similar to the case presented here.

[22] Acknowledgments. We would like to thank the WEC, JSOC, and ESOC teams for continuous support of Cluster operations. The Whisper operations and data analysis are supported by CNES through contract CNES/60015.

[23] Zuyin Pu thanks Scott Boardsen and John Menietti for their assistance in evaluating this paper.

\section{References}

Balogh, A., et al. (2001), The Cluster Magnetic Field Investigation: Overview of in-flight performance and initial results, Ann. Geophys., 19, $1207-1217$.

Canu, P., et al. (2001), Identification of natural plasma emissions observed close to the plasmapause by Cluster-Whisper relaxation sounder, Ann. Geophys., 19, 1697-1709.

Canu, P., P. Décréau, S. Escoffier, and S. Grimald (2006), Observations of continuum radiations close to the plasmapause: Evidence for small scale sources, in Planetary Radio Emissions VI, edited by H. O. Rucker, W. S. Kurth, and G. Mann, pp. 289-298, Austrian Acad. of Sci. Press, Vienna.

Décréau, P. M. E., P. Fergeau, V. Krasnoselskikh, M. Lévêque, P. Martin O. Randriamboarison, F. X. Sené, J. G. Trotignon, P. Canu, and P. B. Mögensen (1997), WHISPER, a resonance sounder and wave analyser: Performances and perspectives for the Cluster mission, Space Sci. Rev, 79, 157-193, doi:10.1023/A:1004931326404.

Décréau, P. M. E., et al. (2001), Early results from Whisper instrument on Cluster: An overview, Ann. Geophys., 19, 1241-1258.

Décréau, P. M. E., et al. (2004), Observation of continuum radiations from the CLUSTER fleet: First results from direction finding, Ann. Geophys., 22, 2607-2624

Etcheto, J., P. J. Christiansen, M. P. Gough, and J. G. Trotignon (1982), Terrestrial continuum radiation observations with GEOS-1 and ISEE-1, Geophys. Res. Lett., 9, 1239-1242, doi:10.1029/GL009i011p01239.

Farrell, W. M. (2001), Direct generation of O-mode emission in a dense, warm plasma: Applications to interplanetary type II emissions and others in its class, J. Geophys. Res., 106(A8), 15,701-15,709, doi:10.1029/ 2000JA000156

Farrell, W. M., M. L. Kaiser, W. S. Kurth, M. D. Desch, D. A. Gurnett, G. B. Hospodarsky, and R. J. MacDowall (2004), Remote sensing of possible plasma density bubbles in the inner Jovian dayside magnetosphere, J. Geophys. Res., 109, A09S14, doi:10.1029/2003JA010130.

Gough, M. P. (1982), Non-thermal continuum emissions associated with electron injections: Remote plasmapause sounding, Planet. Space Sci., 30, 657-668, doi:10.1016/0032-0633(82)90026-5.

Gough, M. P., P. J. Christiansen, G. Martelli, and J. Gershuny (1979), Interaction of electrostatic waves with warm electrons at the geomagnetic equator, Nature, 279, 515-517, doi:10.1038/279515a0.

Gurnett, D. A. (1975), The Earth as a radio source: The nonthermal continuum, J. Geophys. Res., 80, 2751-2763, doi:10.1029/ JA080i019p02751.

Gurnett, D. A., and L. A. Frank (1976), Continuum radiation associated with low-energy electrons in the outer radiation zone, J. Geophys. Res., 81, 3875-3885, doi:10.1029/JA081i022p03875.

Gustafsson, G., et al. (1997), The electric field and wave experiment for the Cluster mission, Space Sci. Rev., 79, 137-156, doi:10.1023/ A:1004975108657.

Hubbard, R. F., and T. J. Birmingham (1978), Electrostatic emissions between electron gyroharmonics in the outer magnetosphere, J. Geophys. Res., 83, 4837-4850.

Kasaba, Y., H. Matsumoto, K. Hashimoto, R. R. Anderson, J. L. Bougeret, M. L. Kaiser, X. Y. Wu, and I. Nagano (1998), Remote sensing of the plasmapause during substorms: Geotail observation of nonthermal continuum enhancement, J. Geophys. Res., 103, 20,389-20,405, doi:10.1029/98JA00809.

Kurth, W. S. (1992), Continuum radiation in planetary magnetospheres, in Planetary Radio Emission III, edited by H. O. Rucker, S. J. Bauer, and M. L. Kaiser, pp. 329-350, Austrian Acad. of Sci. Press, Vienna.

Kurth, W. S., D. A. Gurnett, and R. R. Anderson (1981), Escaping nonthermal continuum radiation, J. Geophys. Res., 86, 5519-5531, doi:10.1029/JA086iA07p05519.

Menietti, J. D., and P. H. Yoon (2006), Plasma waves and fine structure emission bands within a plasmapause density cavity source region, Geophys. Res. Lett., 33, L15101, doi:10.1029/2005GL025610.

Morgan, D. D., and D. A. Gurnett (1991), The source location and beaming of terrestrial continuum radiation, J. Geophys. Res., 96, 9595-9613, doi:10.1029/91JA00314.

Rönnmark, K. (1985), Generation of magnetospheric radiation by decay of Bernstein waves, Geophys. Res. Lett., 12, 639-642, doi:10.1029/ GL012i010p00639.

Weatherwax, A. T., J. Labelle, M. L. Trimpi, R. A. Treumann, J. Minow, and C. Deehr (1995), Statistical and case studies of radio emissions observed near $2 f_{c e}$ and $3 f_{c e}$ in the auroral zone, J. Geophys. Res., 100, 7745-7757, doi:10.1029/94JA03147

Willes, A. J., D. Bale, and Z. Kuncic (1998), A $z$ mode electron-cyclotron maser model for bottomside ionosphere harmonic radio emissions, J. Geophys. Res., 103, 7017-7026, doi:10.1029/97JA03601.

Yoon, P. H., A. T. Weatherwax, and T. J. Rosenberg (1996), Lower ionospheric cyclotron maser theory: A possible source of $2 f_{c e}$ and $3 f_{c e}$ auroral radio emissions, J. Geophys. Res., 101, 27,015-27,025, doi:10.1029/ 96JA02664.

Yoon, P. H., A. T. Weatherwax, and T. J. Rosenberg (1998), On the generation of auroral radio emissions at harmonics of the lower ionospheric electron cyclotron frequency: $X, O$ and $Z$ mode maser calculations, J. Geophys. Res., 103, 4071-4078, doi:10.1029/97JA03526.

P. Canu, Centre d'Etude des Environnements Terrestre et Planétaires, 10-12 avenue de l'Europe, F-78140 Vélizy-Villacoublay, France.

P. M. E. Décréau, A. Rochel, and X. Vallières, Laboratoire de Physique et Chimie de l'Environnement, CNRS, 3A avenue de la Recherche Scientifique, F-45071 Orléans, France.

S. Grimald, Department of Space and Climate Physics, Mullard Space Science Laboratory, University College London, Surrey RH5 6NT, UK. 